\title{
Spectroscopic observations of pulsations in roAp stars
}

\author{
M. Sachkov and T. Ryabchikova \\ Institute of Astronomy, Russian Academy of Science, 48 Pyatnitskaya str., \\ 119017 Moscow, Russia
}

\begin{abstract}
Spectacular progress in the observational study of rapidly oscillating Ap (roAp) stars has been achieved by considering high time- and spectral-resolution spectroscopy in addition to the classical high-speed photometric measurements. Such observations led to the discovery of a multitude of unexpected phenomena, generally pointing to an extreme vertical chemical non-uniformity of the atmospheres of magnetic CP stars. A detailed analysis of spectroscopic pulsational behaviour allows us to establish a relationship between pulsations and the vertical stratification of the chemical elements. This has become possible with the use of a Very Large Telescope on relatively bright stars. Using Extremely Large Telescopes promises farther heights for asteroseismology.
\end{abstract}

Keywords. Stars: pulsation - Stars: atmospheres - Stars: roAp

\section{Introduction}

The rapidly oscillating Ap (roAp) stars are close to the main sequence and within the edges of the classical instability strip. They were discovered photometrically by Kurtz (1982). These stars have a strong magnetic field and strong overabundances of rare-earth elements (REE). The relatively rich p-mode spectra of the roAp stars makes these objects attractive for asteroseismology studies.

\section{Spectroscopic study}

Light variations in the roAp stars have very low amplitudes. But the first spectroscopic observations revealed large RV amplitudes up to $1 \mathrm{kms}^{-1}$ and even more. The most surprising characteristic of the RV pulsations in roAp stars is their selectivity. The highest RV amplitudes are observed in the lines of the rare-earth elements (REE) (Savanov, Malanushenko \& Ryabchikova 1999), while spectral lines of the other elements are constant or weakly pulsating. This pulsational behavior is typical for all roAp stars observed spectroscopically up to now.

Another characteristic of the RV pulsations in roAp stars - a phase shift between RV variations in the lines of different elements/ions - was first detected by Kochukhov \& Ryabchikova (2001) in $\gamma$ Equ and was later confirmed for other stars (HD 166473 by Kurtz, Elkin \& Mathys (2003), HD 24712 by Sachkov et al. (2004)). Pulsation curves for the lines of the same REE ions differ in phase, and larger gradual shifts occur between RV curves of different REE elements. These spectacular new observational results come from high time resolution high spectral resolution and high signal-to-noise ratio data that only the largest telescopes can provide. 


\section{Line formation depth and pulsations}

Recently we found a relationship between pulsations and vertical stratification of chemical elements (Sachkov et al. 2005) for the roAp star HD 24712. We calculated the depths of the line forming regions for Nd, hydrogen and Ca based on an LTE stratification study. The RV curves for several ions and the optical depth of the line forming region are shown in Fig. 1. These results allow us to construct a stratified model of the atmosphere of this roAp star.

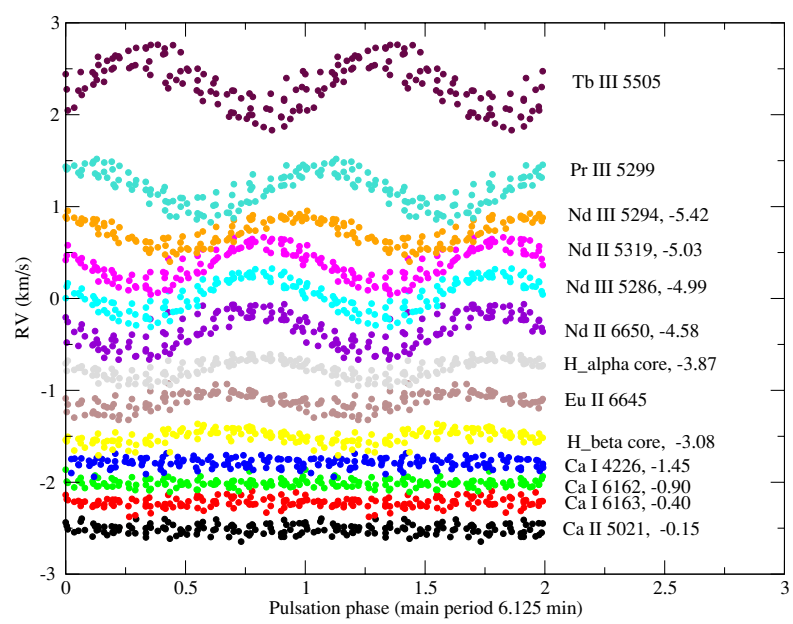

Figure 1. The RV curves for selected lines and depths of the line forming regions (next to the wavelengths) for roAp star HD 24712.

\section{Conclusions}

Recent spectacular progress in roAp star pulsation studies was achieved by using high time and spectral resolution observations. Further stratification analysis of rare-earth elements, especially prosiodimium and therbium, is highly desirable. Such an analysis, in addition to the pulsation one, will provide extremely important information about the distribution of the pulsations through the stellar atmosphere.

\section{Acknowledgements}

This work was supported by the RFBR grants 04-02-16788 and by the grant of the RF president MK-954.2005.2.

\section{References}

Kochukhov, O. \& Ryabchikova, T. 2001, A\& A 377, L22

Kurtz, D.W. 1982, MNRAS 200, 807

Kurtz, D.W., Elkin, W.G. \& Mathys, G. 2003, MNRAS 343, L5

Sachkov, M., Ryabchikova, T., Kochukhov, O., Weiss, W.W., Reegan, P. \& Landstreet, J.D. 2004, in: D.W. Kurtz \& K.R. Pollard (eds.) Variable Stars in the Local Group, ASP Conf. Ser. Vol. 310, San Francisco ASP, p. 208

Sachkov, M., Ryabchikova, T., Bagnulo, S. et al. 2005, Mem. S. A. It. in press

Savanov, I.S., Malanushenko, V.P. \& Ryabchikova, T.A. 1999, Astron. Lett. 25, 902 